\title{
The influence of substrate material on the resistance of composite films based on reduced graphene oxide and polystyrene
}

\author{
M. N. Nikolaeva ${ }^{1}$, E. V. Gushchina ${ }^{2}$, M. S. Dunaevskii ${ }^{2}$, A. T. Dideikin ${ }^{2}$, A. N. Bugrov ${ }^{1,3}$, T. D. Anan'eva ${ }^{1}$ \\ ${ }^{1}$ Institute of macromolecular compounds RAS, Bolshoy pr. 31, 199004 St. Petersburg, Russian Federation \\ ${ }^{2}$ Ioffe Institute RAS, Politekhnicheskaya ul. 26, 194021 St. Petersburg, Russian Federation \\ ${ }^{3}$ Saint Petersburg Electrotechnical University "LETI", \\ ul. Professora Popova 5, 197376 St. Petersburg, Russian Federation \\ marianna_n@mail.ru*,katgushch@yandex.ru, Mike.Dunaeffsky@mail.ioffe.ru, \\ dideikin@mail.ioffe.ru, alexander.n.bugrov@gmail.com, anthracene@hq.macro.ru
}

PACS 36.20.-r; 68.65.Pq; 71.20.Rv; 72.80. Tm; 74.78.-w

DOI 10.17586/2220-8054-2017-8-5-665-669

Current and surface topographies of composite based on polystyrene with reduced graphene oxide were investigated using atomic force microscopy. Different substrates such as gold, silicon and graphite were used for this purpose. The strong influence of the substrate's nature on the current distribution map $I(x, y)$ and the current-voltage characteristics was observed. This effect can be related to different adhesion of composite on the investigated substrates.

Keywords: reduced graphene oxide, polystyrene, composite, resistance, atomic force microscopy.

Received: 16 September 2017

Revised: 9 October 2017

\section{Introduction}

There are many studies devoted to the conductivity of graphene oxide in polymer composites [1-8]. Graphene oxide is a dielectric, but with appropriate treatment its band gap can be decreased to zero $[9,10]$. In this regard, the conductivity of the reduced graphene oxide (RGO) varies from that of a semiconductor to a metal. This can be one of the reasons for the discrepancies in the conductivity values of graphene-containing polymer composites, which are fixed by the authors. Also, not only the chemical and thermal pre-history of graphene inclusions affects the conductivity of polymer composites, but also the introduction approach, distribution uniformity and adhesion at the polymer-graphene interface play an important role. For example, it was shown in [11] that the conductive properties of a polystyrene composite with RGO (named in this paper as "graphene flakes") are significantly dependent on the dispersion degree and homogeneity distribution of the filler in the polymer matrix. The filler localization near the surface of the polymer films leads to high conductivity in the case of styrene copolymerization with graphene functionalized by vinyl groups. It is worth noting that RGO functionalized by 3-(trimethoxysilyl)propyl methacrylate can form covalent bonds with styrene under predetermined polymerization conditions, similar to the work [12]. At the same time, the mechanical mixing of the modified RGO with polymer matrix, as well as the copolymerization of styrene with unmodified filler, give weakly conducting compositions.

A large number of articles devoted to graphene/polymer or RGO/polymer composites have shown that their conductivity is proportional to the filler content. At the same time, there are articles where very small amounts of graphene $(0.01 \mathrm{wt} . \%)$ contribute to the appearance of high conductivity $[13,14]$ and even superconductivity $[15,16]$. In our case, the object of study is the composites demonstrating superconducting features based on polystyrene with RGO combining graphene fragments and oxidized groups. Thus, the goal of this article is to measure resistance values of polystyrene/RGO films and to determine the influence of substrate on the composite conductivity. We suppose that various substrates will provide different adhesion degree for the RGO/polystyrene composite. Since polystyrene is dielectric itself, for any changes in the conductivity under the influence of the substrate material, one can expect RGO inclusions only. It is important to mention that electron tunneling is possible if the distance between electron states is less than $2 \mathrm{~nm}$, thus the conductivity of composite film is limited by this distance. It is important because the key point of getting the charge into the composite film is its electrification by the substrate metal even with a simple metal/polymer contact [17]. We assume the adhesion degree can correlate with the amount (area) of conducting regions on the composite film along with the conductivity value. The conductive atomic force microscopy (CAFM) method was used for detailed study of the conductivity of composite films on various substrates. Previously, it was shown by CAFM analysis of polystyrene/RGO thin films that high-conductivity regions exhibit ohmic current-voltage characteristics, starting with weak electric fields [13]. In 
addition, this method allowed that non-conducting regions to become conductive when a threshold pre-destructive electric field was reached. The effect can be reversible, when the electric field is reduced below the threshold value. The reverse switching to a non-conducting state occurs by analogy with results of A. N. Ionov et.al. [18].

\section{Experimental}

Natural crystalline graphite was used as an initial material for GO production. Modified Hummers wet chemical method was used to produce graphene oxide [10,19]. After series of chemical reactions in liquid medium, prepared RGO was extracted from an aqueous suspension by drying at room temperature in air. Synthetic details were described previously $[13,14]$. Surface modification of RGO by 3-(trimethoxysilyl)propyl methacrylate was carried out using the method presented in our previous work [11]. Synthesis of polymer-inorganic composite based on polystyrene with surface-modified particles of RGO was performed by in-situ polymerization in solution. We put $0.5 \mathrm{ml}$ of styrene, $0.5 \mathrm{ml}$ of toluene and $5 \mathrm{mg}$ of $2,2^{\prime}$-azobis(2-methylpropionitrile) as an initiator into an ampule containing $5 \mathrm{mg}$ of RGO (1 wt.\%). Then, the ampule with the reaction mixture was purged with argon for $10 \mathrm{~min}$, sealed and sonicated. Polymerization was carried out for 35 hours at $70{ }^{\circ} \mathrm{C}$, every hour for 15 minutes the ampule was sonicated. The content of the covalently bound RGO in the composite was about $0.01 \mathrm{wt} . \%$.

Graphite and silicon plates have been used as substrates, as well as a vacuum deposited gold coating on glass. All substrates had a surface roughness not exceeding several nanometers, which was confirmed by the AFM data. Atomic force microscope Solver P47-Pro was used for surface and current topography measurements. The surfaces of the electrodes before the composite film deposition were thoroughly cleaned with acetone and ethanol. Films of the composite were deposited on electrodes through the dispenser by casting from $1 \%$ polymer solution in toluene. The films' thicknesses were evaluated from concentration of composite solution and surfaces profiles of substrate electrodes as well were controlled by the interference microscope according to the method described in [20]. Thus, the thicknesses of investigated films were approximately $2 \mu \mathrm{m}$. The diamond AFM tip doped by nitrogen with curvature radius $50 \mathrm{~nm}$ was used as an upper electrode in CAFM measurements. The Ohm's nature of obtained current-voltage characteristics exclude the presence of a breakdown in films which have been investigated.

\section{Results and discussion}

The obtained RGO/polystyrene composite exhibited a different level of resistance depending on the lower electrode nature, according to CAFM measurements. The composite does not form percolation cluster in our case, since the concentration threshold of graphene is much higher, $0.9 \mathrm{wt} . \%$ [7]. Therefore, conductivity is possible only on areas containing RGO inclusions.

The surface topography (a) of the polymer composite on gold substrate and the current distribution (b) taken at the voltage value at the electrodes $U=3 \mathrm{~V}$ are shown in Fig. 1. Here, as well as on the following figures, more protruding parts of the surface and the largest currents are colored by white in grayscale palette. It can be seen that there is a correlation between the topography of the surface and the current distribution. The greatest currents are observed in places protruding above the surface and associated with the inclusion of RGO in the polymer matrix. The resistance value $(R)$ on the gold coating is $5 \times 10^{6} \Omega$ for used probe geometry of the atomic force microscope [21]. If measurements are made on electrodes with a larger contact area $(S)$, the resistance of the polymer composite will decrease in proportion to the increase in $S$. For example, the calculated value resistance will be $\sim 1 \Omega$ for macroscopic electrodes with a contact area of $\sim 400 \mu \mathrm{m}^{2}$.

The topography of the composite surface on silicon (a), as well as the current distribution (b) on it at a voltage $U=3 \mathrm{~V}$ is shown in Fig. 2(a). The current-voltage characteristic of the most conductive region of composite film on a silicon substrate and the silicon one are shown in Fig. 3. These current-voltage characteristics demonstrate non-liner behavior that may indicate the Schottky barrier presence in this case. Fig. 4 shows the composite surface topography on graphite (a), as well as the current distribution on it (b) taken at the voltage value of the electrode $U=1 \mathrm{~V}$.

The dark areas in the Fig. 1(b), Fig. 2(b), and Fig. 4(b) correspond to low conductivity. Current distribution maps $I(x, y)$ were taken at voltages not exceeding the value of $10^{6} \mathrm{~V} / \mathrm{cm}$ to exclude electrical breakdown of the film.

There is a correlation between the topography of the surface and the current distribution: a current exceeding the noise is observed only at those points which dominate the nearest surrounding surface. A similar correlation between surface topography and current distribution is observed, even when the polymer was deposited onto other metal substrates. Moreover, the current-voltage characteristics at the points demonstrating high conductivity obey Ohm's law (Fig. 5). In addition, forward and reverse motions of the current-voltage characteristics have also been reproduced. 

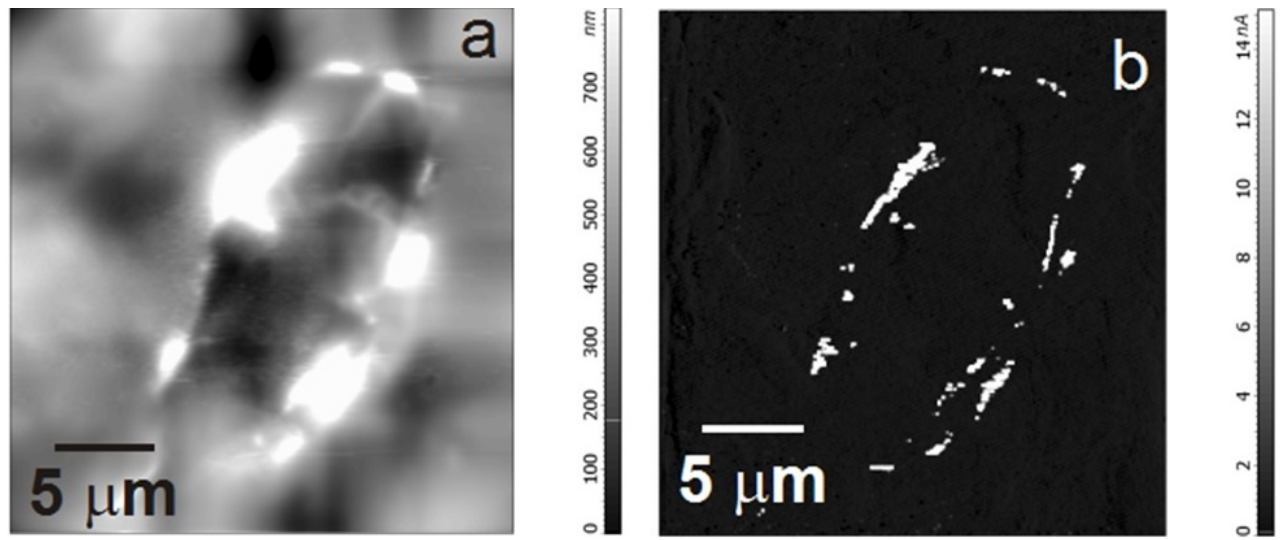

FIG. 1. Surface (a) and current (b) topography of a composite film deposited on a gold substrate at voltage of $3 \mathrm{~V}$
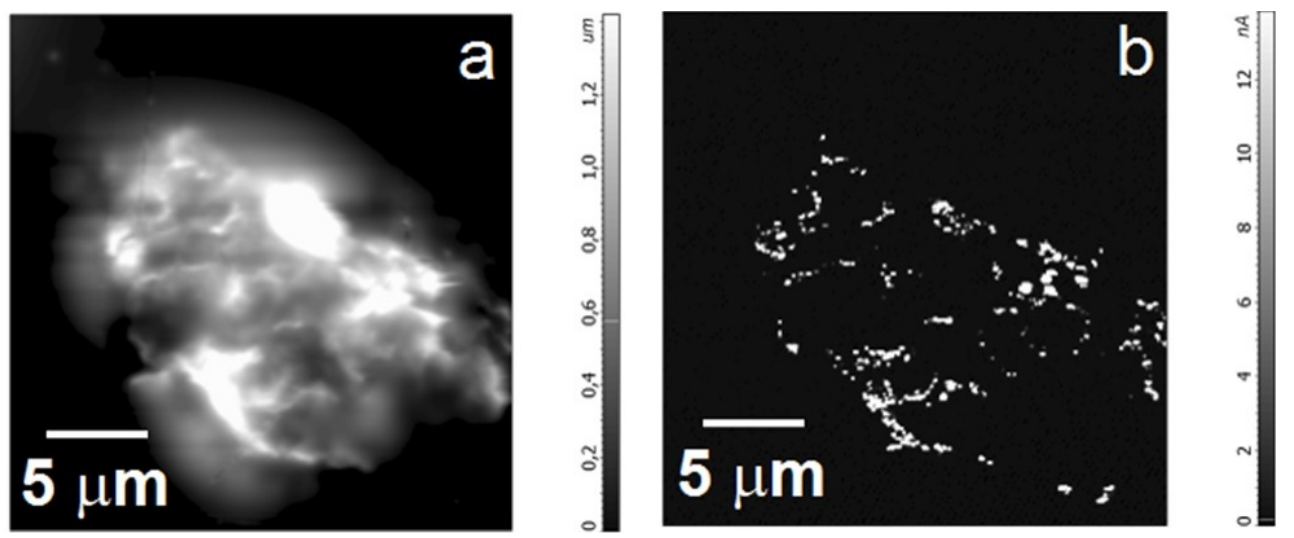

FIG. 2. Surface (a) and current (b) topography of a composite film deposited on silicon substrate at voltage of $3 \mathrm{~V}$

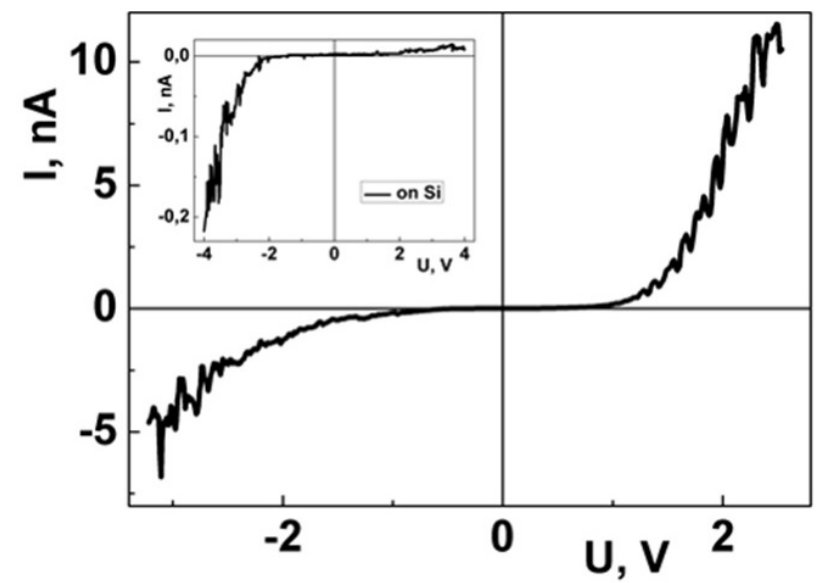

FIG. 3. Current-voltage characteristics of the conductive area of composite film on silicon substrate and the current-voltage characteristics of silicon (in insertion) 

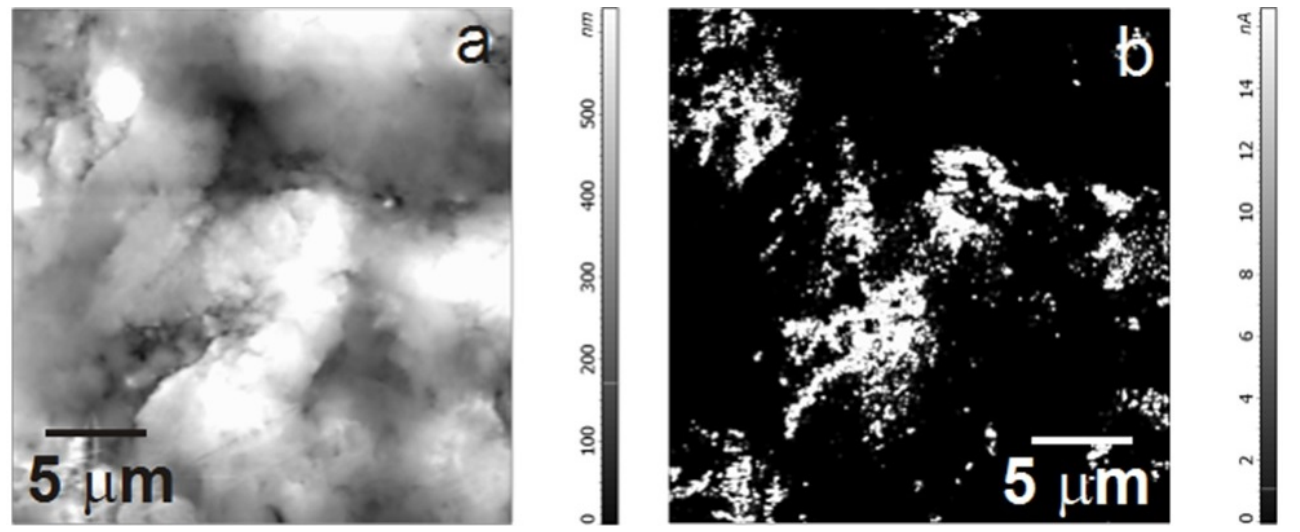

FIG. 4. Surface (a) and current (b) topography of composite film deposited on graphite substrate at a voltage of $1 \mathrm{~V}$

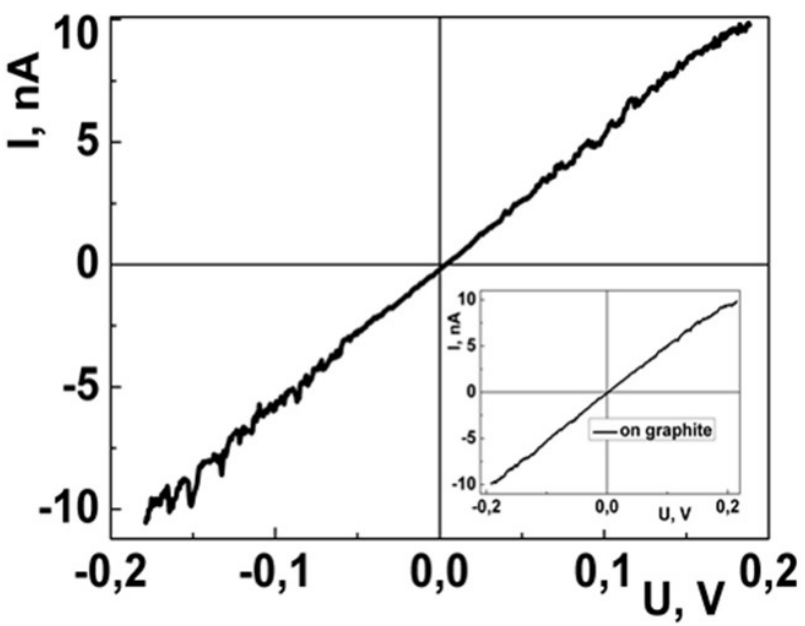

FIG. 5. Current-voltage characteristic of the conductive area of composite film on graphite substrate (a) and the current-voltage characteristic of graphite (in insertion)

Despite gold's high conductivity, the RGO/polystyrene resistance values on gold turned out to be comparable to the values for the composite film on silicon (Fig. 1(b), 2(b)) and amounted to approximately $2 \times 10^{8} \Omega$. This result can be explained by weak adhesion of the RGO particles to the gold substrate. Thus, the resistance of the film conductive areas related to RGO is higher than the resistance of gold by more than one and a half orders of magnitude. The resistance of gold in our case is $5 \times 10^{6} \Omega$, as was indicated above. It is also important to note that conductive areas for films on gold are much less than for films on graphite, in which the measured resistance of the composite conducting inclusions was of $3 \times 10^{7} \Omega$. This is almost an order of magnitude lower than the RGO resistance on the gold substrate. The graphite substrate is obviously the most suitable material for achieving the high-conductivity state for polystyrene-based RGO composite films. The results obtained for the resistance and the conductive areas of composite films are probably related to the fact that both styrene rings and graphene inclusions are close in chemical nature to the graphite, creating the greatest surface adhesion. This facilitates the creation of numerous conducting channels through the composite film from the one electrode to the other. At the same time, the gold substrate for our type of composite does not promote good adhesion and gives high resistance values. One of the factors which improve adhesion, film contact force with silicon substrate and its conductivity obviously is 3-(trimethoxysilyl)propyl methacrylate.

It can be concluded that there is no relation between the conductivity of the RGO/polystyrene composite and the electron work function of substrate material, as was shown in [22]. For example, gold has an electron work function of about $4.7 \mathrm{eV}$. Thus, viewed composite films exhibit a low resistance on a gold substrate according to [22]. In fact, we see in present work the inverse situation.

The conductive area resistance of $\mathrm{RGO} /$ polystyrene film on a graphite substrate at room temperature is approximately 2 orders of magnitude lower, than its resistance on gold, and is equal to the neat graphite resistance. 
In addition to the semiconductor values of conductivity that are typical for RGO at room temperature, the metallic type of conductivity is observed in composites with decreasing temperature to that of liquid nitrogen [13], as well as superconducting properties when their thickness is about $150 \mathrm{~nm}[15,16]$. This unusual character for the temperature dependences of resistance requires theoretical explanations. The authors of [23] propose that sufficiently high density of states for such systems as considered $\mathrm{RGO}$ /polystyrene composite results in the high temperature of either spin ordering or superconducting pairing.

\section{Conclusions}

Films of RGO/polystyrene composite which demonstrate superconducting features under special conditions were investigated by the CAFM method. The experiments showed that the density of the conductive areas on the surface of the polymer films with the same thicknesses and the absolute values of the current intensity depend on the substrate's nature, as well as the applied electric field at the metal/polymer contact area. The minimum resistance of the RGO/polystyrene film areas was $3 \times 10^{7} \Omega$ when using this tip geometry and under the conditions of the best adhesion on graphite, which approximately corresponds to the graphite resistance.

\section{Acknowledgements}

We are grateful to Alexander N. Ionov and to Leonid N. Ionov (Ioffe Institute) for useful discussion and for gold substrate preparation. A. T. Dideikin acknowledges RFBR, grant 15-02-05153, for support.

\section{References}

[1] Rani A., Nam S., et.al. Electrical conductivity of Chemically reduced graphene powders under compression. Carbon Lett., 2010, 11(2), P. 90-95

[2] Neustroev E.P., Nogovitsyna M.V., Solovyova Yu.S., et al. Study of electrical conductivity of thermally reduced graphene oxide. RENSIT, $2015,7(2)$, P. $162-167$.

[3] Chen Y., Fu K., Zhu S., et al. Reduced graphene oxide films with ultrahigh conductivity as Li-ion battery current collectors. Nano Lett., 2016, 16(6), P. 3616-3623.

[4] Jungo S.T., Oh S.H., Kim H.B., et. al. The optical and electrical properties of graphene oxide with water-soluble conjugated polymer composites by radiation. J. Nanosci. Nanotechnol., 2013, 13(11), P. 7358-7364.

[5] Nirmala R., Navamathavan R., Kim H.Y., Park S.J. Electrical properties of conductive nylon66/graphene oxide composite nanofibers. $J$. Nanosci. Nanotechnol., 2015, 15(8), P. 5718-22.

[6] Chamingkwan P., Matsushita K., Taniike T., Terano M. Enhancement in mechanical and electrical properties of polypropylene using graphene oxide grafted with end-functionalized propylene. Materials, 2016, 9, P. 240-253.

[7] Syurik Ju., Alyabyeva N., Alekseev A., Ageev O.A. AFM-based model of percolation in graphene-based polymer nanocomposites. Composite Science and Technology, 2014, 95, P. 38-43.

[8] Song M. Graphene functionalization and its application to polymer composite materials. Nanomat. and en., 2013,2 , P. 97-111.

[9] Mikoushkin V.M., Shnitov V.V., Nikonov S.Yu. et al. Controlling graphite oxide bandgap width by reduction in hydrogen. Tech. Phys. Lett., 2011, 37(10), P. 942-945.

[10] Aleksenskii A.E., Brunkov P.N., Dideikin A.T. et al. Single-layer graphene oxide films on a silicon surface. Tech. Phys., 2013, 58(11), P. $1614-1618$

[11] Nikolaeva M.N., Anan'eva T.D., Bugrov A.N. et.al. Correlation between structure and resistance of composites based on polystyrene and multilayered grapheme oxide. Nanosystems: physics, chemistry, mathematics, 2017, 8(2), P. 266-271.

[12] Yevlampieva N., Bugrov A., Anan'eva T., et al. Soluble poly (methyl methacrylate) composites containing covalently associated zirconium dioxide nanocrystals. Am. J. Nano Res. and Appl., 2014, 2(2), P. 1-8.

[13] Khairullin A.R., Nikolaeva M.N., Bugrov A.N. Resistance of the composite films based on polystyrene and graphene oxide. Nanosystems: physics, chemistry, mathematics, 2016, 7(6), P. 1055-1058.

[14] Nikolaeva M.N., Bugrov A.N., et al. Conductive properties of the composite films of graphene oxide based on polystyrene in a metalpolymer-metal structure. Russ. J. Appl. Chem., 2014, 87(8), P. 1151-1155.

[15] Ionov A.N. Josephson current-voltage characteristic of a composite based on polystyrene and graphene oxide. Tech. Phys. Lett., 2015, 41(7), P. 651-653.

[16] Ionov A.N. Josephson-Like Behaviour of the Current-Voltage Characteristics of Multi-graphene Flakes Embedded in Polystyrene. J. Low Temp. Phys., 2016, 185(5-6), P. 515-521.

[17] Duke C.B., Fabish T.J. Charge-induced relaxation in polymers. Phys Rev Lett., 1976, 37, P. $1075-1078$.

[18] Ionov A.N., Nikolaeva M.N., Rentzsch R. Local distribution of high-conductivity regions in polyamide thin films. JETP Letters, 2007, 85(12), P. 636-638.

[19] Hummers W., Offeman R. Preparation of graphitic oxide. J. Am. Chem. Soc., 1958, 80(6), P. 1339-1339.

[20] Nikolaeva M., Boiko Y., Martynenkov A. Supramolecular structure and conductive properties of dielectric polymers in metal/polymer/metal systems. Int. J. Polym. Mater., 2013, 62(13), P. 706-710.

[21] Ionov A.N., Nikolaeva M.N., Rentzsch R. Local distribution of high-conductivity regions in polyamidine thin films. JETP Letters, 2007, 85(12), P. 636-638.

[22] Ionov A.N., Dunaevskii M.S., Nikolaeva M.N., et al. The dependence of polymer conductivity on the work function of metallic electrodes. Ann. Phys., 2009, 18(12), P. 959-962.

[23] Volovik G.E., Pudalov V.M. Graphite on graphite. JETP Letters, 2016, 104(12), P. 880-882. 